\title{
Identification of 6-demethoxy-6-methylgeldanamycin and its implication of geldanamycin biosynthesis
}

\author{
Siyang $\mathrm{Ni}^{1}$, Bingya Jiang ${ }^{1}$, Linzhuan Wu, Yiguang Wang, Hongxia Zhou, Weiqing He, Hongyuan Wang, \\ Jianhua Zhu, Shufen Li, Ting Li and Kan Zhang
}

The Journal of Antibiotics (2014) 67, 183-185; doi:10.1038/ja.2013.94; published online 9 October 2013

Keywords: 6-demethoxy-6-methylgeldanamycin; geldanamycin biosynthesis; Streptomyces hygroscopicus 17997

Geldanamycin (1, Figure 1), the first member of benzoquinone ansamycins, was isolated from Streptomyces hygroscopicus in $1970 .{ }^{1}$ Although exhibiting potent cytotoxicity against various cancer cells, 1 is not a clinical compound due to its severe hepatotoxicity and poor water solubility. ${ }^{2}$ 17-AAG (17-allylamino-17-demethoxygeldanamycin) as a semisynthetic derivative of 1 with much improved water solubility is currently under clinical trial for breast cancer treatment. ${ }^{3}$ Many new analogs or derivatives of $\mathbf{1}$ have been created or discovered in the past few years. ${ }^{4-8}$

We are interested in natural 1 analogs and understanding their synthetic mechanisms. We identified such analogs as 4,5-dihydro-4hydroxygeldanamycins, thiazinogeldanamycin and 19-S-methylgeldanamycin from S. hygroscopicus 17997 and characterized their synthetic mechanisms. ${ }^{9-13}$ We also discovered a minor component 7-descarbamoyl-7-hydroxygeldanamycin from a $g d m N$ disruption mutant of S. hygroscopicus 17997, which presented an additional proof for C-7 carbamoylation taking place before C-4,5 oxidation in $\mathbf{1}$ biosynthesis. ${ }^{14}$

Recently, as a result of our continued efforts for natural $\mathbf{1}$ analogs, we discovered 6-demethoxy-6-methylgeldanamycin (2) in 1 preparation from S. hygroscopicus 17997. In this paper, we reported the structure of 2 and its implication of $\mathbf{1}$ biosynthesis.

Some preparations of $\mathbf{1}$ were found to contain $\mathbf{1}$ analogs as small or trace impurities. ${ }^{15,16}$ The HPLC of our 1 preparation (with a purity of about $90 \%$; see Supplementary material: a brief description of 1 preparation from S. hygroscopicus 17997) from S. hygroscopicus 17997 displayed a small peak at $24.7 \mathrm{~min}$ (about $1.7 \%$ of the principle 1 peak at $22.3 \mathrm{~min}$; Figure 2). The peak revealed a molecular ion at $\mathrm{m} / \mathrm{z} 567$ $\left([\mathrm{M}+\mathrm{Na}]^{+}\right)$, which exhibited a typical $\mathrm{MS}^{2}$ fragment pattern of 1 (Supplementary Figure S1). The $\mathrm{m} / z 567$ aroused our interests, as we could not assign a reasonable structure for it from MS data and current understanding of $\mathbf{1}$ biosynthesis. ${ }^{17-19}$
To elucidate the structure of the analog with $m / z 567$ (2) by NMR, a total amount of $1070 \mathrm{mg} 1$ preparation, dissolved in $10 \mathrm{ml}$ dimethyl sulfoxide, was used to make a pure preparation of 2 by reversed-phase HPLC (Shimadzu LC-20AP, SHIMADZU, Kyoto, Japan; YMC ODSA, $21.2 \times 150 \mathrm{~mm}$, mobile phase $\mathrm{MeOH}-\mathrm{H}_{2} \mathrm{O}, 62-100 \%$ in $21 \mathrm{~min}$, $12.5 \mathrm{ml} \mathrm{min}^{-1}$, wavelength $254 \mathrm{~nm}$; Supplementary Figure S2). After evaporation, an amount of $5.6 \mathrm{mg}$ of 2 as yellow amorphous powder was obtained. Analytical HPLC indicated that it displayed an UV absorption profile very similar to that of 1 (Supplementary Figure S3).

The molecular formula of 2 was established as $\mathrm{C}_{29} \mathrm{H}_{40} \mathrm{~N}_{2} \mathrm{O}_{8}$ by HR-ESI $(+)$-MS $\quad(m / z \quad 567.26596$, calculated 567.26769 for $\mathrm{C}_{29} \mathrm{H}_{40} \mathrm{~N}_{2} \mathrm{O}_{8} \mathrm{Na}$, Supplementary Figure $\mathrm{S} 4$ ), which is one oxygen atom less than $1\left(\mathrm{C}_{29} \mathrm{H}_{40} \mathrm{~N}_{2} \mathrm{O}_{9}\right)$. The ${ }^{1} \mathrm{H}$ and ${ }^{13} \mathrm{C}$ NMR spectra of 2 (Supplementary Figures S5 and S6) were very similar to those of $\mathbf{1}^{18}$ Comparison of the NMR data of $\mathbf{2}$ with those of $\mathbf{1}$ revealed that the only difference between the two compounds was replacement of the 6 -methoxy group in 1 by the 6-methyl group in 2 , which was confirmed by the $2 \mathrm{D}$ NMR data analysis of 2 . In particular, the ${ }^{1} \mathrm{H}-{ }^{1} \mathrm{H}$ COSY correlations of $\mathrm{H}-5 / \mathrm{H}-6 / \mathrm{H}-7, \mathrm{H}-6 / \mathrm{H}_{3}-23$ and $\mathrm{HMBC}$ correlations of $\mathrm{H}_{3}-23 / \mathrm{C}-4, \mathrm{C}-6, \mathrm{C}-5$, in combination with the shifts of these proton and carbon resonances established the $\mathrm{CH}_{3}-6$ in 2 . Therefore, the structure of 2 was determined to be 6-demethoxy-6methylgeldanamycin (Figure 1). The NMR chemical shifts of 2 were assigned completely by HSQC, COSY and HMBC spectroscopic data (Supplementary Figures S7-S10) as indicated in Table 1.

Compound $\mathbf{2}$ is a shunt product in $\mathbf{1}$ biosynthesis. Compound 1's biosynthesis consists of a starter unit (3-amino-5-hydroxybenzoic acid) assembly, extender units (one malonyl, two 2-methoxymalonyl and four 2-methylmalonyl units for polyketide chain building) condensation and tailoring modifications. ${ }^{17-19}$ Obviously, 2 is derived from mis-incorporation of a 2-methylmalonyl unit in place

Key Laboratory of Biotechnology of Antibiotics of Ministry of Health, Institute of Medicinal Biotechnology, Chinese Academy of Medical Sciences and Peking Union Medical College, Beijing, China

${ }^{1}$ These authors contributed equally to this work.

Correspondence: Professor L Wu, Key Laboratory of Biotechnology of Antibiotics of Ministry of Health, Institute of Medicinal Biotechnology, Chinese Academy of Medical Sciences and Peking Union Medical College, Tiantan Xili, Beijing 100050, China.

E-mail: wulinzhuan@imb.pumc.edu.cn

Received 21 May 2013; revised 7 August 2013; accepted 27 August 2013; published online 9 October 2013 

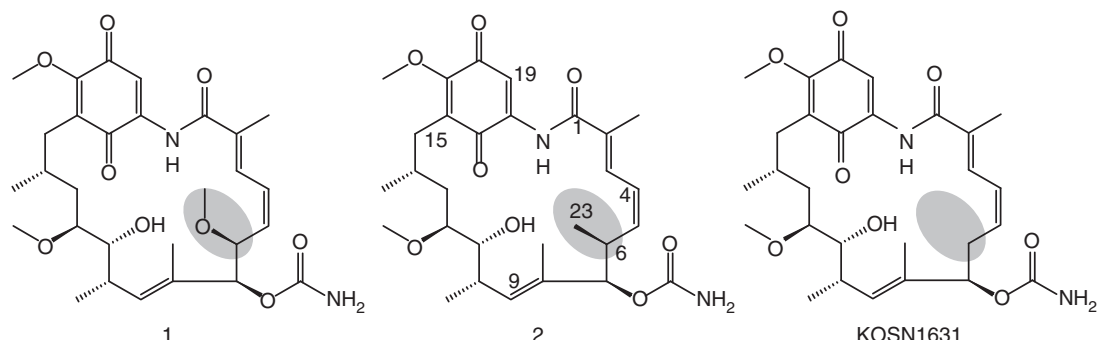

Figure 1 Chemical structure of 1, 2 and KOSN1631. A full color version of this figure is available at The Journal of Antibiotics journal online.

- XWC of DAD Spectral Data:

300.0 to $308.0 \mathrm{~nm}$ from Sample 1 (HPLCMS) of GDM (recalibrated) (recalibrated). wiff

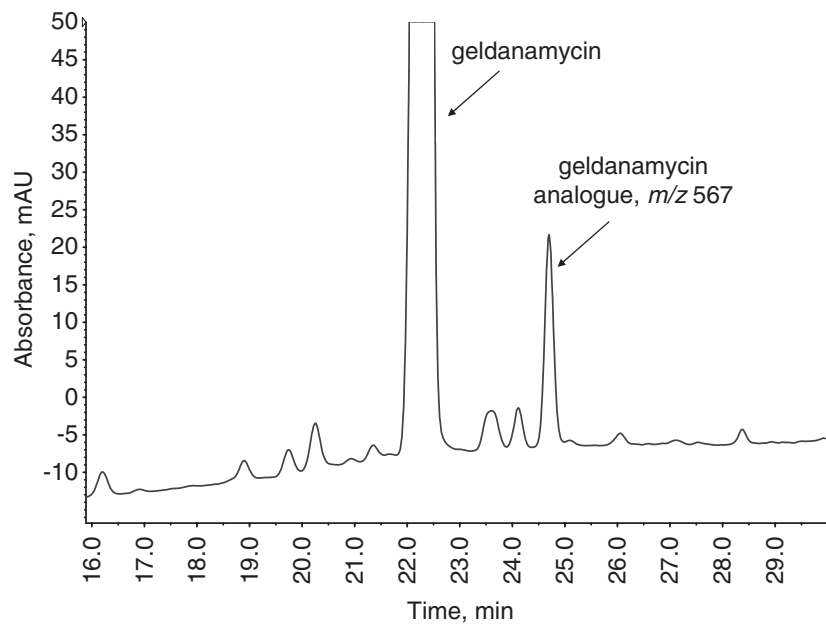

Figure 2 HPLC of geldanamycin (1) preparation from S. hygroscopicus 17997. HPLC parameters: Agilent 1200 RRLC system (Agilent, Waldbronn, Germany); Dikma Diamonsil $C_{18}$ column $(4.6 \times 150 \mathrm{~mm}, 5 \mu \mathrm{m}$, DIKMA, Beijing, China), mobile phase $\mathrm{MeOH}-\mathrm{H}_{2} \mathrm{O}, 40-100 \%$ in $30 \mathrm{~min}$, $1.0 \mathrm{ml} \mathrm{min}-1$, wavelength $304 \mathrm{~nm}$. A full color version of this figure is available at The Journal of Antibiotics journal online.

of normal incorporation of a 2-methoxymalonyl unit into the polyketide chain in $\mathbf{1}$ biosynthesis. It is interesting to note that macbecin (or ansamitocin) as a close $\mathbf{1}$ analog from Actinosynnema pretiosum ATCC 31280 (or 31565) also contains a methyl (not a methoxyl) side group at C-6 of its polyketide chain, indicating that a 2-methylmalonyl unit incorporates into the polyketide chain in the corresponding condensation reaction of macbecin (or ansamitocin) biosynthesis. $^{20,21}$

Compound 2 must share the same absolute configurations with $\mathbf{1}$, as $\mathbf{2}$ is co-produced with $\mathbf{1}$ and must be biosynthesized by the same set of enzymes as $1 .{ }^{22-24}$ In particular, the incorporation of 2-methylmalonyl (not 2-methoxymalonyl) unit into the polyketide chain for 2 biosynthesis should not change the configuration of C-6, which is presumably determined by the enoylreductase domain of module 5 of polyketide synthases (PKS) for 1 biosynthesis. ${ }^{22}$

The identification of $\mathbf{2}$ in $\mathbf{1}$ preparation indicates that the acyltransferase domain of module 5 (AT5) of PKS for 1 biosynthesis shows a promiscuous substrate specificity for 2-methoxymalonyl CoA and, to a small degree, 2-methylmalonyl CoA. This phenomenon is observed occasionally in the biosynthesis of some microbial polyketides. For examples, the two components (A and B) of galbonolide are derived from substrate tolerance (for 2-methoxymalonyl and 2-methylmalonyl CoA) of AT5 of PKS in galbonolide biosynthesis. ${ }^{25}$
Table 1 The NMR spectra data of 2

\begin{tabular}{|c|c|c|}
\hline \multirow[b]{2}{*}{ Position } & \multicolumn{2}{|r|}{2} \\
\hline & $\delta_{C}$ & $\delta_{H}(J$ in $H z)$ \\
\hline 1 & 168.7 & - \\
\hline 2 & 132.9 & - \\
\hline 3 & 128.6 & 7.02, d (11.4) \\
\hline 4 & 122.4 & $6.30, \mathrm{dd}(11.4,10.8)$ \\
\hline 5 & 142.4 & $5.83, \mathrm{dd}(10.8,8.4)$ \\
\hline 6 & 39.3 & $2.96, \mathrm{dq}(8.4,6.6)$ \\
\hline 7 & 81.2 & $5.29, \mathrm{~s}$ \\
\hline 8 & 133.3 & - \\
\hline 9 & 131.1 & $5.62, d(9.6)$ \\
\hline 10 & 32.5 & $2.70, \mathrm{~m}$ \\
\hline 11 & 73.1 & $3.49, \mathrm{~m}$ \\
\hline 12 & 81.0 & $3.40, \mathrm{~m}$ \\
\hline 13 & 34.6 & 1.77, m 1.65, overlap \\
\hline 14 & 28.6 & 1.66 , overlap \\
\hline 15 & 32.2 & 2.45 , overlap 2.43 , overlap \\
\hline 16 & 127.7 & - \\
\hline 17 & 156.9 & - \\
\hline 18 & 184.2 & - \\
\hline 19 & 111.3 & $7.25, \mathrm{~s}$ \\
\hline 20 & 138.3 & - \\
\hline 21 & 184.9 & - \\
\hline 22 & 12.4 & $1.99, \mathrm{~s}$ \\
\hline 23 & 14.2 & $1.09, \mathrm{~d}(6.6)$ \\
\hline 24 & 156.1 & - \\
\hline 25 & 13.3 & $1.68, \mathrm{~s}$ \\
\hline 26 & 12.9 & $0.98, d(6.6)$ \\
\hline 27 & 56.5 & $3.34, \mathrm{~s}$ \\
\hline 28 & 22.8 & $0.96, d(7.2)$ \\
\hline 29 & 61.6 & $4.11, \mathrm{~s}$ \\
\hline $1-\mathrm{NH}-$ & - & $8.80, \mathrm{~s}$ \\
\hline 7-OCONH & - & 4.70, brs \\
\hline $11-\mathrm{OH}$ & - & $2.84, d(5.4)$ \\
\hline
\end{tabular}

${ }^{1} \mathrm{H}$ and ${ }^{13} \mathrm{C}$ NMR spectra data $(\delta)$ were obtained at 600 and $125 \mathrm{MHz}$, respectively, on INOVA501 with tetramethylsilane as internal standard, and measured in $\mathrm{CDCl}_{3}$ at room temperature.

The two components (A and B) of epothilone are also derived from relaxed substrate specificity (for malonyl and 2-methylmalonyl CoA) of acyltransferase domain of module 3 of PKS in epothilone biosynthesis. $^{26}$ Recently, three rapamycin analogs (as impurities) were reported, with each one resulted from mis-incorporation of a 2-ethylmalonyl unit (in place of normal incorporation of a 2-methylmalonyl unit) into the polyketide chain at corresponding positions by the acyltransferase domain of module 3, 7 or 13 of PKS in rapamycin biosynthesis. ${ }^{27}$ 
To date, it is still difficult to foretell whether an acyltransferase domain in modular PKS possesses promiscuous substrate specificity or not, or even to predict its substrate specificity for 2-methoxymalonyl CoA, because only a few such acyltransferase domains are reported. We believe that the AT5 of PKS for $\mathbf{1}$ biosynthesis may be useful in establishing an in silico method to predict substrate promiscuity of acyltransferase domains of modular PKS in the future.

Patel et al. ${ }^{28}$ reported an 1 analog 6-desmethoxygeldanamycin (KOSN1631, Figure 1) produced by an AT5-engineered strain of S. hygroscopicus, in which the AT5 of 1 PKS was replaced with the acyltransferase domain (with a substrate specificity for malonyl CoA) of module 2 of rapamycin PKS by genetic recombination. Compared with 1, KOSN1631 showed a significant decrease in cytotoxicity against human breast adenocarcinoma cell line $\mathrm{SKBr} 3$, with an $\mathrm{IC}_{50}$ value of $3.2 \mu \mathrm{M}$ (for $1,0.041 \mu \mathrm{M}$ ). We conducted a preliminary cytotoxicity assay of $\mathbf{2}$ against human liver hepatocellular carcinoma cell line HepG2 by MTT (3-(4,5-dimethylthiazol-2-yl)-2,5-diphenyltetrazolium bromide). ${ }^{29}$ Compound 2 also exhibited lower cytotoxicity than 1 against $\mathrm{HepG} 2$, with an $\mathrm{IC}_{50}$ value of $10.5 \mu \mathrm{M}$ (for $1,0.37 \mu \mathrm{M}$ ), suggesting that the 6-methoxy group may have an important role in 1's cytotoxicity against cancer cells.

\section{ACKNOWLEDGEMENTS}

This work was supported by National Natural Science Foundation of China (81172964), National Mega-project for Innovative Drugs (2012ZX09301002001-016, 2012ZX09301002-003) and the Fundamental Research Funds for the Central Universities (2012N09).

1 DeBoer, C., Meulman, P. A., Wnuk, R. J. \& Peterson, D. H. Geldanamycin, a new antibiotic. J. Antibiot. (Tokyo) 23, 442-447 (1970).

2 Fukuyo, Y., Hunt, C. R. \& Horikoshi, N. Geldanamycin and its anti-cancer activities. Cancer Lett. 290, 24-35 (2010).

3 Gartner, E. M. et al. A phase II study of 17-allylamino-17-demethoxygeldanamycin in metastatic or locally advanced, unresectable breast cancer. Breast Cancer Res. Treat 131, 933-937 (2012)

4 Eichner, S., Floss, H. G., Sasse, F. \& Krischning, A. New, highly active nonbenzoquinone geldanamycin derivatives by using mutasynthesis. Chembiochem 10, 1801-1805 (2009).

$5 \mathrm{Wu}, \mathrm{C}$. Z. et al. New non-quinone geldanamycin analogs from genetically engineered Streptomyces hygroscopicus. J. Antibiot. (Tokyo) 64, 461-463 (2011).

6 Eichner, S. et al. Broad substrate specificity of the amide synthase in S. hygroscopicus - new 20-membered macrolactones derived from geldanamycin. J. Am. Chem. Soc. 134, 1673-1679 (2012).

7 Wu, C. Z. et al. Enzymatic glycosylation of non-benzoquinone geldanamycin analogs via Bacillus UDP-glycosyltransferase. Appl. Environ. Microbiol. 78, 7680-7686 (2012).
8 Wu, C. Z., Jang, J. H., Ahn, J. S. \& Hong, Y. S. New geldanamycin analogs from Streptomyces hygroscopicus. J. Microbiol. Biotechnol. 22, 1478-1481 (2012).

$9 \mathrm{Ni}, \mathrm{S}$. Y. et al. Thiazinogeldanamycin, a new geldanamycin derivative produced by Streptomyces hygroscopicus 17997. J. Microbiol. Biotechnol. 21, 599-603 (2011).

10 Lin, L. et al. Novel 4,5-dihydro-thiazinogeldanamycin in a gdmP mutant strain of Streptomyces hygroscopicus 17997. Biosci. Biotechnol. Biochem. 75, 2042-2045 (2011).

$11 \mathrm{Liu}, \mathrm{X}$. et al. A pair of sulfur-containing geldanamycin analogues, 19-S-methylgeldanamycin and 4,5-dihydro-19-S-methylgeldanamycin, from Streptomyces hygroscopicus 17997. J. Antibiot. (Tokyo) 64, 519-522 (2011).

$12 \mathrm{Li}, \mathrm{S}$. F. et al. Methanethiol as a catabolite of methionine provides methylthio- group for chemical formation of 19-S-methylgeldanamycin and 17,19-dimethylthioherbimycin A. J. Antibiot. (Tokyo) 66 (8), 499-503.

$13 \mathrm{Li}$, T. et al. Identification of 4,5-dihydro-4-hydroxygeldanamycins as shunt products of geldanamycin biosynthesis. J. Nat. Prod 75, 1480-1484 (2012).

$14 \mathrm{Ni}$, S. Y. et al. 7-O-descarbamoyl-7-hydroxygeldanamycin, a minor component from the $\mathrm{gdmN}$ disruption mutant of Streptomyces hygroscopicus 17997. J. Antibiot. (Tokyo) 63, 623-625 (2010).

$15 \mathrm{Ni}, \mathrm{S}$. Y. et al. Analysis of geldanamycin analogues in trace amounts by LC-MS/MS. Chin. J. Biotechnol. 25, 847-853 (2009).

$16 \mathrm{Zhu}$, J. et al. Preliminary discrimination and assortment of the components in the crude geldanamycin preparation by HPLC-ESI- MS ${ }^{n}$. Chin. J. Antibiot. 36, 207-213 (2011).

17 Rascher, A. et al. Cloning and characterization of a gene cluster for geldanamycin production in Streptomyces hygroscopicus NRRL 3602. FEMS Microbiol. Lett. 218, 223-230 (2003).

18 Hong, Y. S. et al. Inactivation of the carbamoyltransferase gene refines post-polyketide synthase modification steps in the biosynthesis of the antitumor agent geldanamycin. J. Am. Chem. Soc. 126, 11142-11143 (2004).

19 Shin, J. C. et al. Characterization of tailoring genes involved in the modification of geldanamycin polyketide in Streptomyces hygroscopicus JCM4427. J. Microbiol. Biotechnol. 18, 1101-1108 (2008).

20 Zhang, M. Q. et al. Optimizing natural products by biosynthetic engineering: discovery of nonquinone Hsp90 inhibitors. J. Med. Chem. 51, 5494-5497 (2008).

$21 \mathrm{Yu}, \mathrm{T}$. W. et al. The biosynthetic gene cluster of the maytansinoid antitumor agent ansamitocin from Actinosynnema pretiosum. Proc. Natl Acad. Sci. USA 99, 7968-7379 (2002).

22 Lau, J., Fu, H., Cane, D. E. \& Khosla, C. Dissecting the role of acyltransferase domains of modular polyketide in the choice and stereochemical fate of extender units. Biochemistry 38, 1643-1651 (1999).

23 Keatinge-Clay, A. T. A tylosin ketoreductase reveals how chirality is determined in polyketides. Chem. Biol. 14, 898-908 (2007).

$24 \mathrm{Kwan}, \mathrm{D}$. H. et al. Prediction and manipulation of the stereochemistry of enoylreduction in modular polyketide synthases. Chem. Biol. 15, 1231-1240 (2008).

25 Karki, S. et al. The methoxymalonyl-acyl carrier protein biosynthesis locus and the nearby gene with the $\beta$-ketoacyl synthase domain are involved in the biosynthesis of galbonolides in Streptomyces galbus, but these loci are separate from the modular polyketide synthase gene cluster. FEMS Microbiol. Lett. 310, 69-75 (2010).

26 Petković, H. et al. Substrate specificity of the acyl transferase domains of EpoC from the epothilone polyketide synthase. Org. Biomol. Chem. 6, 500-506 (2008).

27 Kong, F. et al. Isolation and structure of homotemsirolimuses A, B, and C. J. Nat. Prod 74, 547-553 (2011).

28 Patel, K. et al. Engineered biosynthesis of geldanamycin analogs for Hsp90 inhibition. Chem. Biol. 11, 1625-1633 (2004).

29 Mosmann, T. Rapid colorimetric assay for cellular growth and survival: application to proliferation and cytotoxicity assays. J. Immunol. Methods 65, 55-63 (1983).

Supplementary Information accompanies the paper on The Journal of Antibiotics website (http://www.nature.com/ja) 